\title{
Computer Aided Diagnosis in Mammography Microcalcification Analysis
}

\author{
Nadin Jamal Abualroos ${ }^{1 *}$, Norlaili Ahmad Kabir ${ }^{2}$ \\ ${ }^{1}$ Advanced Medical and Dental Institute \\ Universiti Sains Malaysia, Malyaisa \\ ${ }^{2}$ School of Physics \\ Universiti Sains Malaysia, Malyaisa \\ *Corresponding author's email: nadin92jamal [AT] gmail.com
}

\begin{abstract}
----
Objectives: The project aims to resolve missed or overlooked breast cancer lesions particularly due to dense breast structure. Dense breast makes microcalcifications difficult to be detected as both normal breast tissue and microcalcification appear white on mammograms. The carry out the project, a breast phantom was fabricated, and ImageJ software was used to improve the visibility of microcalcification.

Methods: The phantom was fabricated, and the physical properties showed similarity to that of breast tissues. CalciumCarbonate powder simulated microcalcifications. Images were acquired using CR mammography. The images acquired were post processed by ImageJ software through applying thresholding technique to help detection of subtle microcalcifications.

Results: Microcalcifications appearance enhanced by threshold technique. The resultant images showed increasing in the contrast value at $\mathrm{ROI}$ from 3.9, 2.2, and 4.2 to 7.2, 6.8, and 7.6 respectively.

Conclusion: The differences in contrast values were more consequential in areas of low Calcium Carbonate concentration after applying threshold technique.
\end{abstract}

Keywords--- Microcalcification - Mammography - Computer Aided Diagnosis - ImageJ software - breast cancer

\section{INTRODUCTION}

Microcalcifications clusters appear on mammogram as high intensity spots of calcium deposit compared to the background tissues $^{1}$. These spots considered as an important major early sign of breast cancer ${ }^{2}$. Microcalcifications have variety of shapes and diameters, and because of their small sizes as well as their similar density to the surrounding breast tissues; they are sometimes difficult to be detected ${ }^{3}$.The average diameter size of individual microcalcifications is around $0.3 \mathrm{~mm}^{4}$. The breast consists of various amount of fatty, glandular, and connective tissue. Arrangement of these tissues translated to structures of different shapes, scales, and densities. Mammograms are very bright in case of breast involving large amount of glandular tissue, which significantly decreases the microcalcifications visibility ${ }^{5}$. Glandular and fibrous tissues are visualized in mammography as radio-opaque, whereas the adipose tissues visualized as radiolucent according to the differences among the $\mathrm{x}$-ray attenuation properties of the different breast tissues. Therefore, a dense breast that has higher proportion of glandular tissues absorbs higher amounts of radiation than an adipose breast ${ }^{6}$.

Mammograms considered as the standard tool for breast cancer screening ${ }^{7}$. Microcalcifications are visible on mammogram as they exhibit higher X-ray attenuation than the surrounding breast tissue. However, overlapping of tissue structures lead to obscure the visualization of micro-calcifications in mammograms ${ }^{8}$.

Because of the similar appearance of breast background tissues and suspicious microcalcifications. Some parts of the background especially the dense tissue part may be visualized brighter than the microcalcifications. Moreover, low contrast of microcalcification to the background tissue when the intensity of microcalcifications is very close to the background tissue will cause difficulty in the detection process of microcalcifications. Due to that, there is a significant risk that a radiologist may misdiagnose some cases in breast cancer diagnosis. It has been reported that 10-30\% of breast lesions are misinterpreted during routine screening of mammograms ${ }^{5}$. The probability of malignancy in mammography can be determined by using certain categories that are based on the shape and distribution of clustered microcalcifications ${ }^{9}$. 
This work aimed to study the detection of microcalcification clusters which are closely similar to the density of normal breast tissue. Dense breast tissue equivalent phantom was fabricated with embedded microcalcifications. The physical properties of the phantom materials including electron density, effective atomic number, and attenuation properties of the breast phantom. The role of computer aided diagnosis (CAD) in the detection of those microcalcifications was studied using ImageJ software.

\section{MATERIALS AND METHODS}

\subsection{Phantom fabrication}

The development of breast tissue equivalent phantom involves the following steps: preparation of materials to emulate background breast tissue and microcalcifications, physical properties measurements; and finally, to fabricate the breast phantom.

Breast tissue equivalent phantom was fabricated by mixing agar powder with grinded chips wood material to deionized water and cooked under slow heat until the entire agar dissolved. Then the mixture was poured into plastic mould. Clustered microcalcifications were simulated by calcium carbonate powder immersed within the agar mixture. The phantom was fabricated at about $5 \mathrm{~cm}$ thickness.

\subsection{Phantom physical properties}

The desired phantom material closely matches the mass attenuation, effective atomic number of the known breast tissue over the mammography energy range. Attenuation coefficient experiment was performed at Radiation Biophysics Lab School of Physics - USM, Penang, Malaysia. Using: Gamma source Am-241 (45 mCi) - Multichannel analyser (MCA) $\mathrm{NaI}(\mathrm{Tl})$ detector, amplifier, voltage supply, and Computer with Maestro Program. Electron density of each material was calculated from its mass density and its atomic composition according to the formula: $p_{e}=p_{m} \cdot N_{A} \cdot\left(\frac{z}{A}\right)$. where $p_{e}$ is electron density, $p_{m}$ is mass density, $N_{A}$ Avogadro's number, $\mathrm{Z}$ is atomic number and $\mathrm{A}$ is atomic weight. Effective atomic number of each material was calculated based on the elemental composition of each material according to this formula:

$z_{\text {eff }}=\sqrt[2.94]{f_{1}\left(z_{1}\right)^{2.94}+f_{2}\left(z_{2}\right)^{2.94}+f_{3}\left(z_{3}\right)^{2.94}+\cdots}$ where $f_{n}$ : is the fraction of the total number of electrons associated with each element, and $z_{n}$ : is the atomic number of each element ${ }^{10}$.

The density of the materials was determined using this $(D=\omega / V)$ formula. Where the mass of each material was measured by using weight balance device, and the volume was measured by placing the material in water's can in which the amount of water it displaces is equal to that of its volume.

The breast tissue equivalent phantoms were scanned by using Computed Radiology Mammography Model (Planmed Sophie Classic). Energy X-ray beam between $28 \mathrm{kvp}$ and $32 \mathrm{kvp}$ was generated to demonstrate the subtle density differences between normal tissues and microcalcifications. X-ray tubes of mammography systems are equipped with Mo/Mo anode/filter was used in the scanning experiment in the range of $28-32 \mathrm{kvp}$. The X-ray images stored directly onto a computer as digital images.

Elem

ental composition of each material was characterized using an energy dispersive X-ray (EDX) elemental analysis brand FEI, Mode Quanta FEG 650.

\subsection{Image acquisition}

The phantom scanned by Computed Radiology Model (Planmed Sophie Classic). Phantom images were acquired by qualified radiographer at the Advanced Medical and Dental Institution (AMDI), Penang, Malaysia. Images were acquired at two different positions: craniocaudal position at 28 and $32 \mathrm{kv}$ respectively, and mediolateral oblique at $30 \mathrm{kv}$.

\subsection{Post-processing}

Post-processing of the images done by ImageJ software by applying thresholding technique. This technique performed to discover abnormalities in a mammogram by separate high concentrated areas of calcium from the low concentrated regions. Greater threshold value was set to show the brightest areas of the image because this is where the most microcalcifications were located. 


\section{RESULTS}

\subsection{Breast tissue equivalent phantom fabrication}

Agar powder, grinded chips wood, and distilled water mixture used to simulate breast tissue equivalent phantom. Calcium Carbonate powder used to simulate breast microcalcifications.

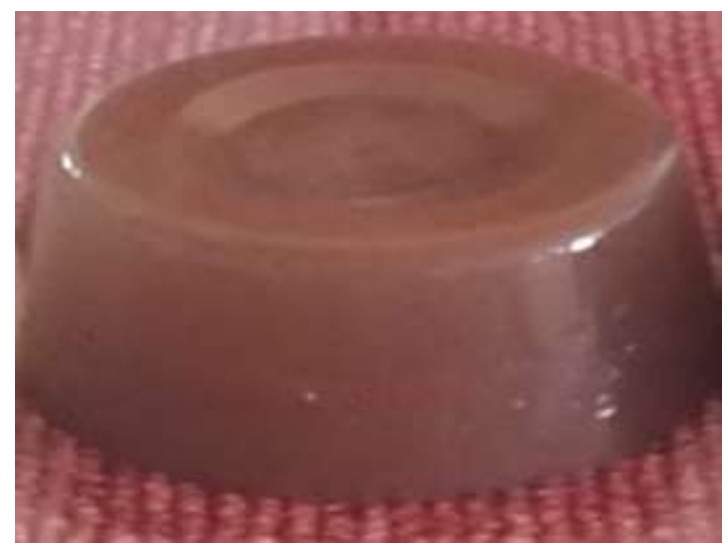

Figure 1. Breast tissue equivalent phantom

\subsection{Phantom radiological and physical properties}

Table 1. phantom materials with their molecular formulas.

\begin{tabular}{lc}
\hline Material & Molecular formula \\
\hline Agar powder & $\mathrm{C}_{14} \mathrm{H}_{24} \mathrm{O}_{9}$ \\
Wood & $\mathrm{C}_{10} \mathrm{H}_{15} \mathrm{O}_{7}$ \\
Calcium carbonate & $\mathrm{CaCO}_{3}$ \\
\hline \hline
\end{tabular}

\subsubsection{Effective atomic number}

3.2.1.1 agar powder

Table 2. agar powder elemental composition.

\begin{tabular}{lll}
\hline Element & Weight $\%$ & $\begin{array}{l}\text { Atomic } \\
\%\end{array}$ \\
\hline C K & 48.73 & 55.93 \\
O K & 50.86 & 43.83 \\
Na K & 0.41 & 0.25
\end{tabular}

Totals $\quad 100.00$ 


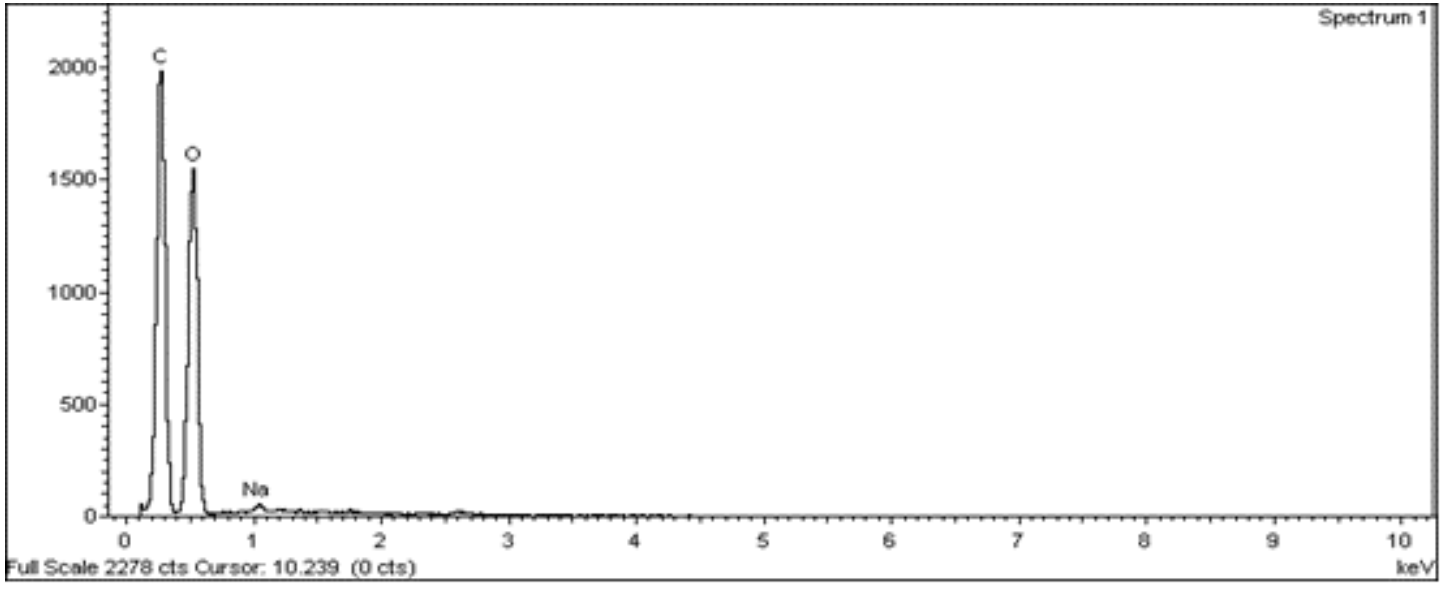

Figure 2. plot of counts versus energy (Kev) for agar powder elemental composition.

Effective atomic number $z_{\text {eff }}=\sqrt[2.94]{0.466(6)^{2.94}+0.133(1)^{2.94}+0.4(8)^{2.94}}=6.725$

\subsubsection{2 wood}

Table 3. wood elemental composition.

\begin{tabular}{lll}
\hline Element & Weight & Atomic \\
& $\%$ & $\%$
\end{tabular}

$\begin{array}{lll}\text { C K } & 51.52 & 58.38 \\ \text { N K } & 3.09 & 3.00 \\ \text { O K } & 45.39 & 38.62\end{array}$

Totals $\quad 100.00$

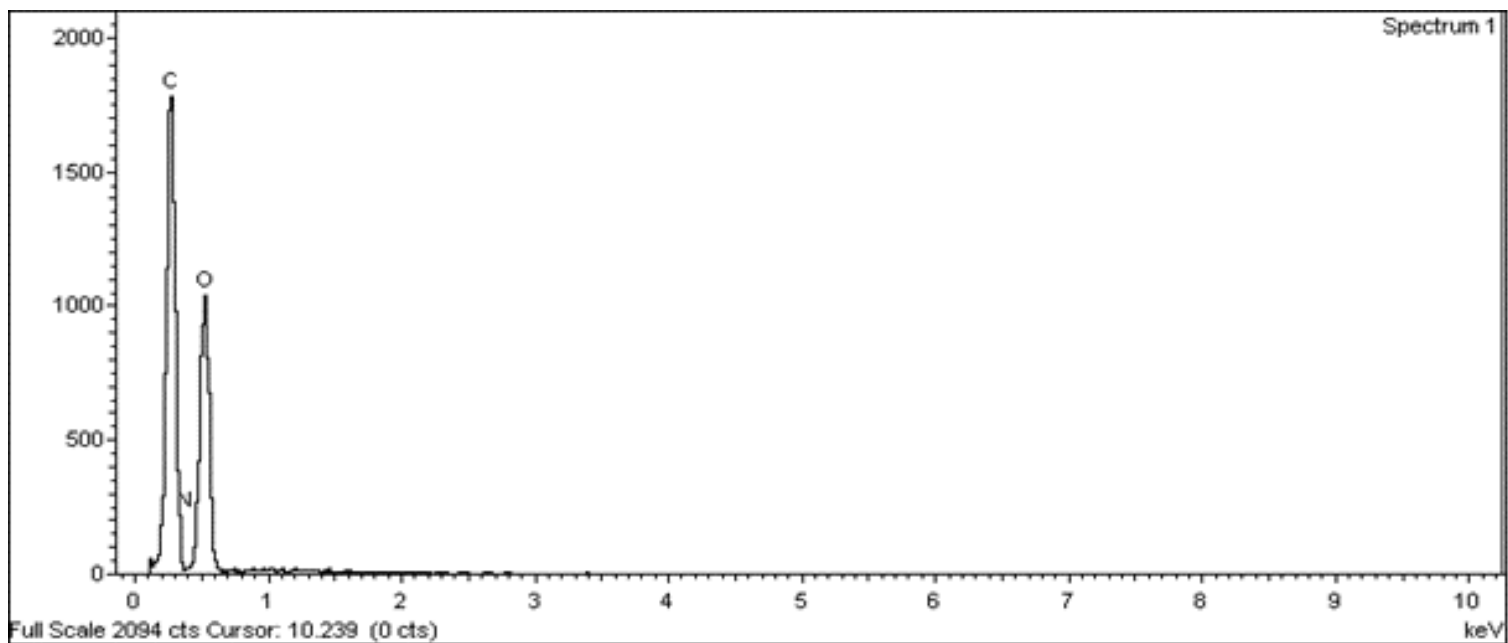

Figure 3. plot of counts versus energy (Kev) for wood elemental composition.

Effective atomic number $z_{\text {eff }}=\sqrt[2.94]{0.458(6)^{2.94}+0.1145(1)^{2.94}+0.4274(8)^{2.94}}=6.8152$ 


\subsubsection{3 calcium carbonate}

Table 4. calcium carbonate elemental composition.

\begin{tabular}{lll}
\hline Element & Weight & Atomic \\
& $\%$ & $\%$
\end{tabular}

$\begin{array}{lll}\mathrm{C} K & 10.81 \quad 18.07\end{array}$

$\begin{array}{lll}\mathrm{N} K & 5.04 \quad 7.22\end{array}$

$\begin{array}{lll}\mathrm{O} K & 43.21 \quad 54.21\end{array}$

$\begin{array}{lll}\text { Ca K } & 40.93 \quad 20.50\end{array}$

Totals $\quad 100.00$

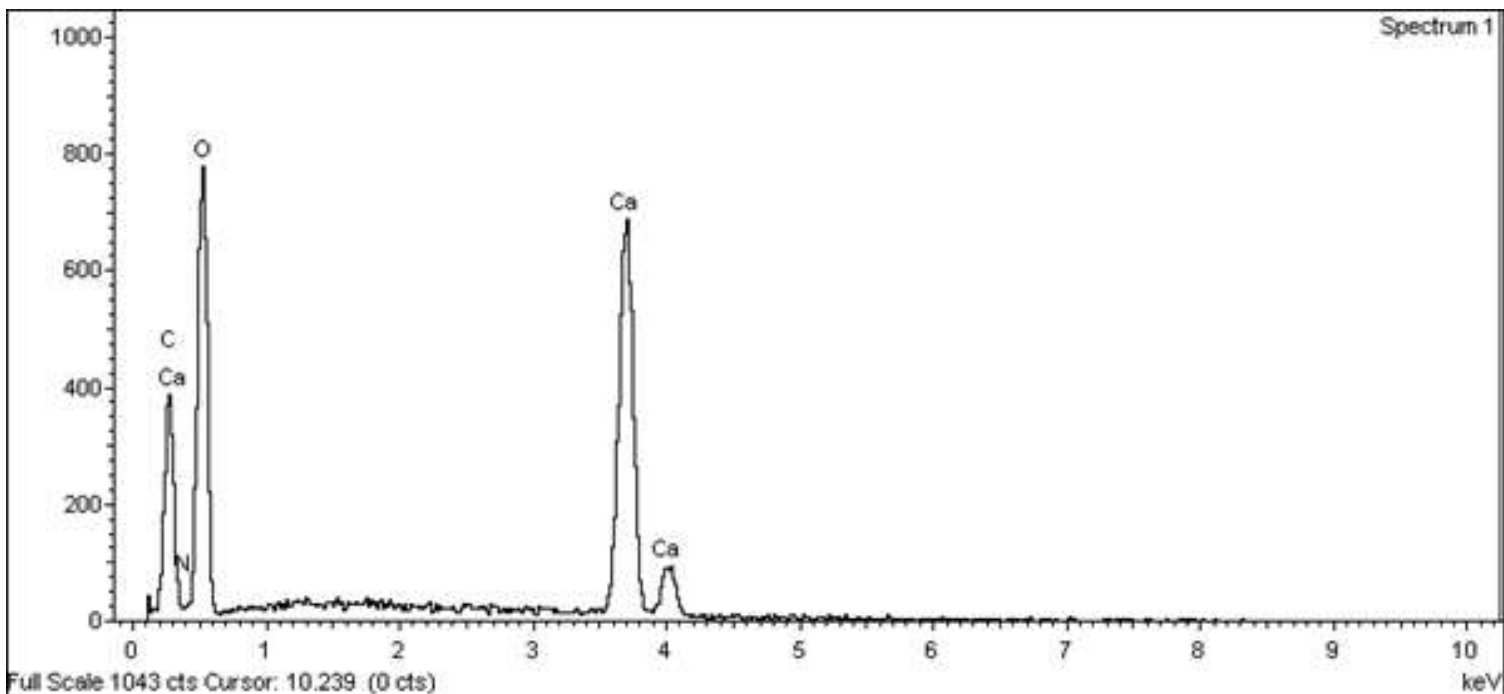

Figure 4. plot of counts versus energy (Kev) for calcium carbonate elemental composition

Effective atomic number $z_{\text {eff }}=\sqrt[2.94]{0.4(20)^{2.94}+0.12(6)^{2.94}+0.48(8)^{2.94}}=15.079$

Table 5. summary of effective atomic numbers for phantom materials.

\begin{tabular}{ll}
\hline Material & Effective atomic number \\
\hline Agar powder & 6.725 \\
Wood & 6.8152 \\
Calcium carbonate & 15.079 \\
\hline \hline
\end{tabular}

\subsubsection{Density}

Weight and volume of each material were measured and using $D=\frac{\omega}{V}$ formula to determine materials density; where $\mathrm{D}$ is density, w is weight, and $\mathrm{v}$ is volume. 
Table 6. Density measurement of the materials.

\begin{tabular}{llll}
\hline \hline & & & \\
\hline Material & Weight $(\mathrm{g})$ & Volume $(\mathrm{ml})$ & Density (w/v) \\
\hline Agar & 7.19 & 5 & 1.43 \\
Wood & 2.31 & 7 & 0.33 \\
Calcium carbonate & 19.11 & 7.5 & 2.548 \\
\hline \hline
\end{tabular}

\subsubsection{Electron density}

Electron density of each material calculated based on its mass density and its atomic composition according to the formula:

$p_{e}=p_{m} \cdot N_{A} \cdot\left(\frac{z}{A}\right)$

Table 7. electron density of the materials

\begin{tabular}{lllll}
\hline \hline Material & \multicolumn{3}{l}{} & \\
& $\begin{array}{l}\text { Mass density } \\
\left(p_{m}\right)\end{array}$ & $\begin{array}{l}\text { Avogadro's } \\
\text { number }\left(N_{A}\right)\end{array}$ & $(\mathrm{Z} / \mathrm{A})$ & Electron density $\left(p_{e}\right)$ \\
\hline Agar & 1.43 & $6.022 \times 10^{23}$ & 2.6192 & $2.255 \times 10^{24}$ \\
Wood & 0.33 & $6.022 \times 10^{23}$ & 2.7532 & $5.471 \times 10^{23}$ \\
Calcium carbonate & 2.548 & $6.022 \times 10^{23}$ & 4.8595 & $7.456 \times 10^{24}$ \\
\hline \hline
\end{tabular}

\subsubsection{Mass attenuation coefficient}

The attenuation of photons when it passes through a medium is described by Lambert beer's law as:

$\mathrm{I}(\mathrm{t})=\mathrm{I}_{\mathrm{O}} \mathrm{e}^{-\mu \mathrm{t}}$, where: $\mathrm{I}_{\mathrm{t}}=$ the intensity of photons transmitted across some distance $\mathrm{x}, \mathrm{I}_{0}=$ the initial intensity of photons, $\mu=$ attenuation coefficient, $\mathrm{t}=$ thickness of the material.

Table 8. mass attenuation coefficient measurements of the materials.

\begin{tabular}{lcccccc}
\hline Material & $\begin{array}{c}\text { Thickness } \\
(\mathrm{mm})\end{array}$ & $\begin{array}{c}\text { Density } \\
(\mathrm{d})\end{array}$ & $\begin{array}{c}\text { Io current } \\
\text { (background }) \\
\mathrm{cps}\end{array}$ & $\begin{array}{c}\text { It } \\
(\mathrm{cps})\end{array}$ & $\begin{array}{c}\text { Attenuation } \\
\text { coefficient } \\
(\mu)\end{array}$ & $\begin{array}{c}\text { Mass } \\
\text { attenuation } \\
\text { coefficient } \\
(\mu / \mathrm{d})\end{array}$ \\
\hline Agar & 1.5 & 1.43 & 6673.53 & 5135.8 & & \\
$\begin{array}{l}\text { Wood } \\
\begin{array}{l}\text { Calcium } \\
\text { carbonate }\end{array}\end{array}$ & 1.1 & 0.33 & 6673.53 & 4014.52 & 0.17461 & 0.1221 \\
\hline \hline
\end{tabular}

\subsection{Image acquisition}

The phantom was scanned to acquire images at craniocaudal and mediolateral oblique positions. 


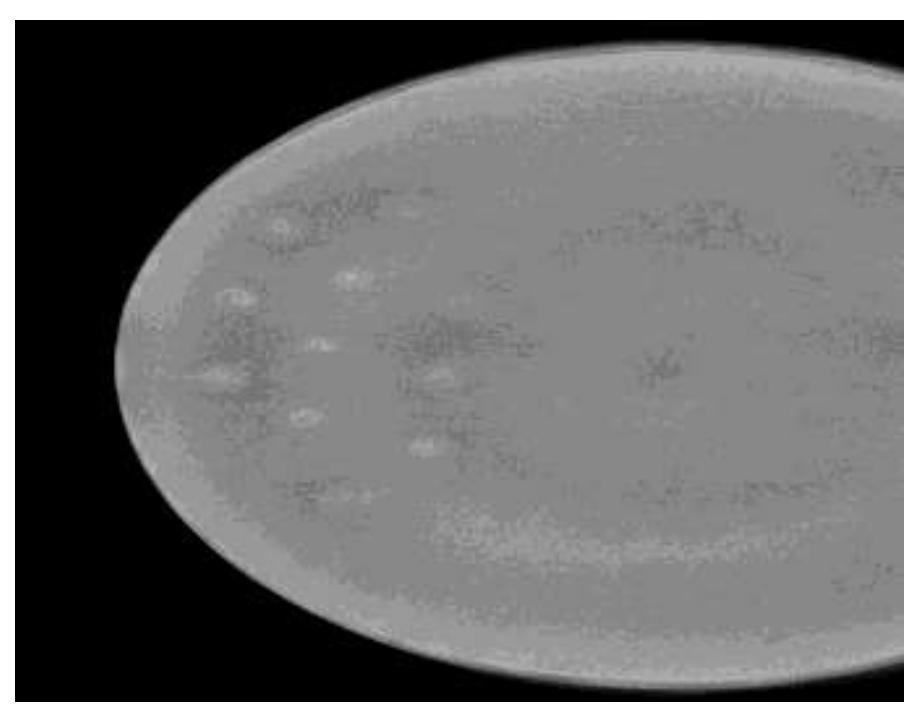

Figure 5. craniocaudal view of the phantom at $28 \mathrm{kv}$

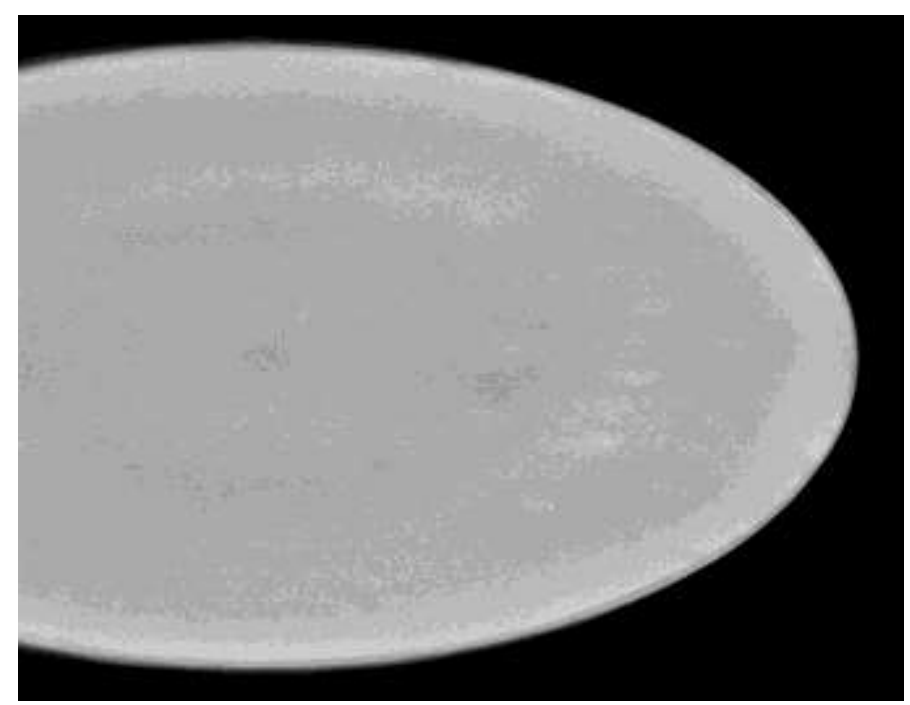

Figure 6. craniocaudal view of the phantom at $32 \mathrm{kv}$

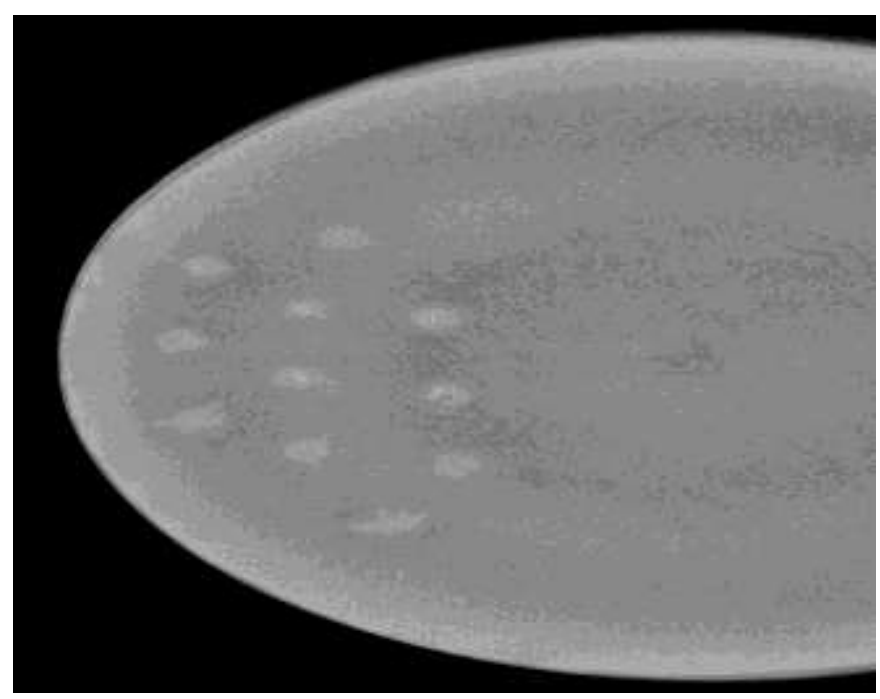

Figure 7. mediolateral oblique view of the phantom at $30 \mathrm{kv}$. 
Cranio-caudal view image acquired at $28 \mathrm{kv}$ showed high contrast difference between normal tissues and microcalcification. However, the image acquired at $32 \mathrm{kv}$ showed less contrast difference between normal tissues and calcifications. As the attenuation differences between tissues is highest at low X-ray energies and is poor at higher energies.

\subsection{Post-processing}

Segmentation plays main role in the detection process of region of interest. Thresholding technique is one of the segmentation methods used which is applied to segment micro-calcifications in mammographic x-ray images. As the areas which containing microcalcifications are somehow inhomogeneous in comparison to the breast tissue.

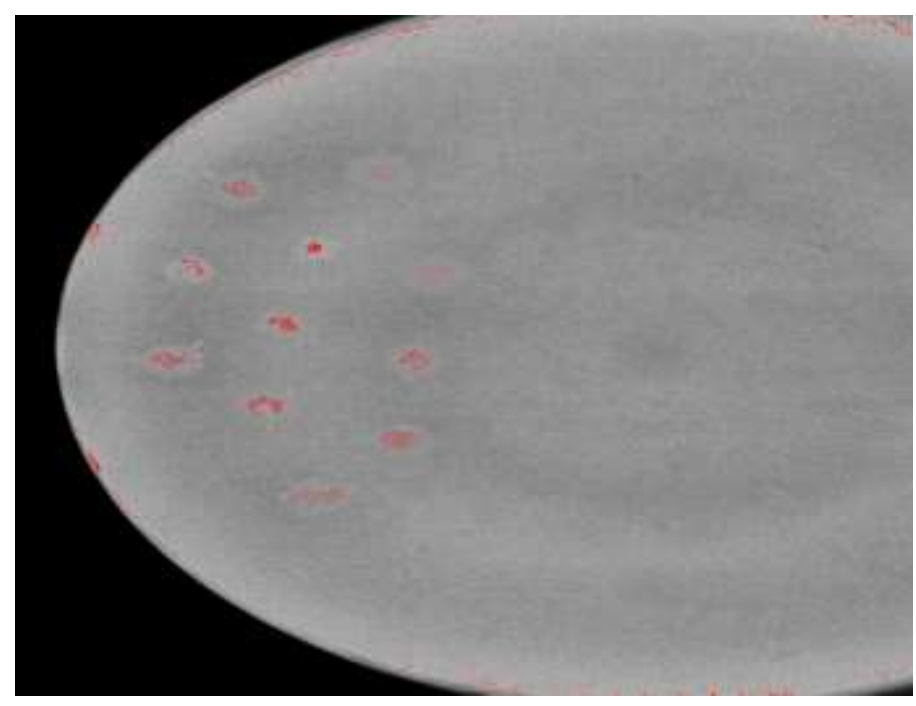

Figure 8. threshold image of the CC view of the phantom at $28 \mathrm{kv}$.

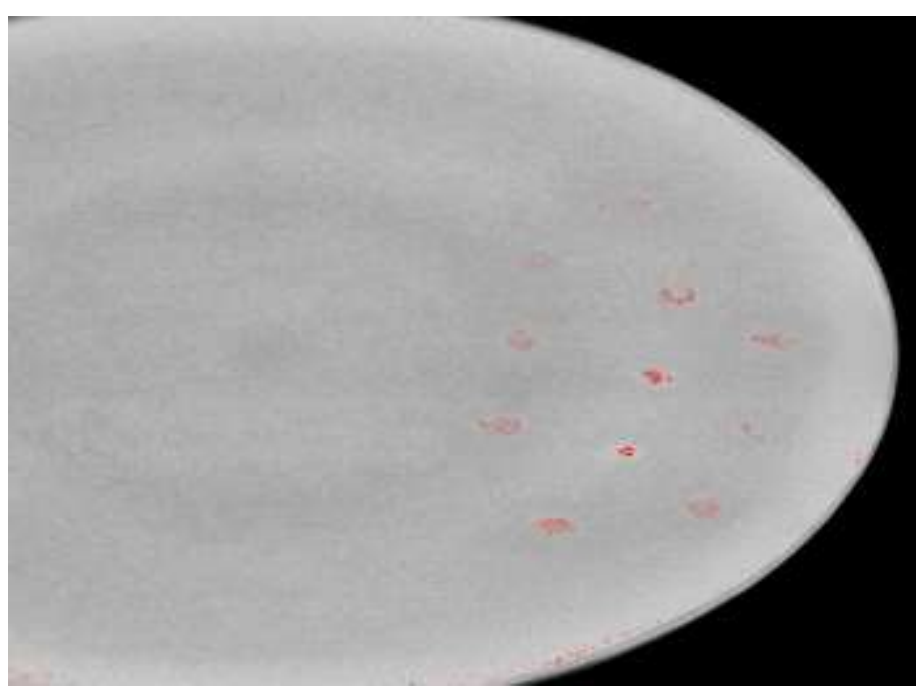

Figure 9. threshold image of the CC view of the phantom at $32 \mathrm{kv}$ 


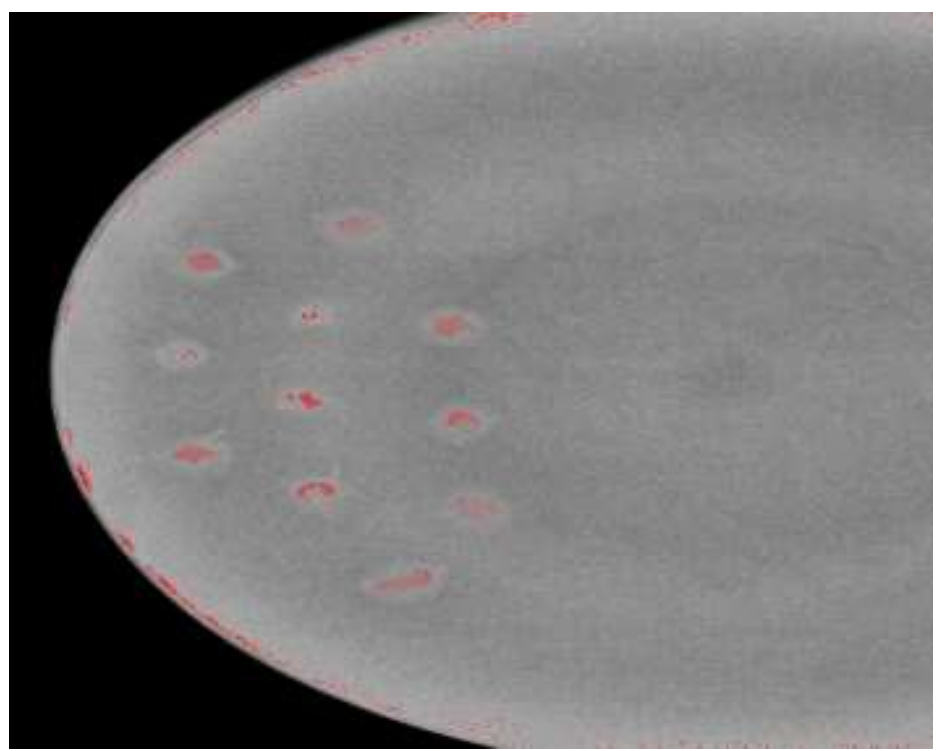

Figure 10. threshold image of MLO view of the phantom at $30 \mathrm{kv}$

Contrast value at region of interest of the acquired images calculated using the following relation, $C N R=$ $\frac{\text { mean of background-mean of ROI }}{\text { standard deviation of background }}$.

Figure 5, 6, And 7. showed the reconstructed image prior to post-processing, the contrast value at ROI is 3.9, 2.2, 4.2 respectively. after applying the thresholding technique, the contrast values increased to 7.2, 6.8, 7.6 respectively.

\section{DISCUSSION}

\subsection{Radiological and physical properties:}

Breast tissue equivalent phantom has been successfully fabricated to closely simulate the physical properties of the breast tissue including effective atomic number, density, electron density, and mass attenuation coefficient.

Effective atomic number of the proposed materials calculated using Murty formula ${ }^{11}$. The results obtained were closely match the values of effective atomic number determined in previous work by M. Kurudirek ${ }^{12}$ having breast tissue effective atomic number of 6.04 which is somehow close to the effective atomic number of agar 6.7 and wood 6.8 .

Density measurement performed showed values of $1.43 \mathrm{~g} / \mathrm{cm} 3$ for agar, $0.33 \mathrm{~g} / \mathrm{cm} 3$ for wood, and $2.548 \mathrm{~g} / \mathrm{cm} 3 \mathrm{for}$ calcium carbonate. According to ICRU-44 report, the density of breast tissue is $1.020 \mathrm{~g} / \mathrm{cm} 3$.

Electron density calculation results of each material was compared to previous work by M. Kurudirek which state that breast tissue electron density is $2.77 \times 10^{23}$

Results of mass attenuation coefficient experiment were compared to the results obtained by ICRU-44 report, that state the mass attenuation coefficient of breast tissue is: $0.1260 \mathrm{~cm}^{2} / \mathrm{g}^{1}$.

\subsection{Image acquisition and post-processing:}

Thresholding technique is one of the segmentation methods used which is applied to segment microcalcifications in mammographic $\mathrm{x}$-ray images. As the areas which containing microcalcifications are somehow inhomogeneous in comparison to the breast tissue. The thresholding eliminates the lower intensity pixels and makes a stronger contrast between the non-cancerous and the cancerous areas.

Post-processing of the images showed increasing in the contrast value at ROI after applying threshold technique values.

Depending on the value of threshold; all pixels less than the threshold are considered as background and the reminder pixels are the breast. According to the results obtained the threshold set was successful to detect most of the calcifications.

\section{CONCLUSION}

Small clusters of micro-calcifications are sometimes appearing within the cancerous areas. Different techniques used to separate the possible cancerous areas from the non-cancerous areas ${ }^{13}$. 
Breast tissue equivalent phantom has been successfully fabricated using agar powder, grinded chips wood and water mixture with embedded Calcium Carbonate powder simulating microcalcification. It was challenging to achieve good result by mixing varied materials having varied physical and radiological properties.

According to previous studies, Calcium Carbonate is a suitable material to simulate microcalcifications. However, it would have been more benefit for this project if egg shells with density of $1.045 \mathrm{~g} / \mathrm{cm}^{3}$ was used instead of Calcium Carbonate, which is an actual limitation of our approach. By measurement performed to obtain the effective atomic number of Calcium Carbonate the experiment shows that the effective atomic number of Calcium Carbonate is around 15, allows occurrence of photoelectric effect interactions. Calcium Carbonate absorbed almost all of the radiation and appeared brighter than background.

Post-processing of the images showed increasing in the contrast value at ROI after applying threshold technique value s. High threshold level selected was successful to detect most of the calcifications.

\section{ACKNOWLEDGMENT}

The support of this work under financial Grant USM RUI GRANT 1001/PFIZIK/811345 by Universiti Sains Malaysia (USM) is gratefully acknowledged.

\section{DISCLOSURE OF CONFLICTS OF INTEREST}

The authors have no relevant conflicts of interest to disclose.

\section{REFERENCES}

1. Quintanilla-dominguez J, Ojeda-magaña B, Cortina-januchs MG, Ruelas R. Sharif University of Technology Image segmentation by fuzzy and possibilistic clustering algorithms for the identification of microcalcifications. Sci Iran. 2011;18(3):580-589. doi:10.1016/j.scient.2011.04.009.

2. Shen L, Rangayyan RM, Desautels JEL. Application of Shape-Analysis to Mammographic Calcifications. IEEE Trans Med Imaging. 1994;13(2):263-274.

3. Wei L, Yang Y, Nishikawa RM, Jiang Y. A study on several machine-learning methods for classification of malignant and benign clustered microcalcifications. IEEE Trans Med Imaging. 2005;24(2):371-380. doi:10.1109/TMI.2004.842457.

4. Li H, Liu KJR, Lo SCB. Fractal modeling and segmentation for the enhancement of microcalcifications in digital mammograms. IEEE Trans Med Imaging. 1997;16(6):785-798. doi:10.1109/42.650875.

5. Wei L, Yang Y, Nishikawa RM. Microcalcification classification assisted by content-based image retrieval for breast cancer diagnosis i. 2009;42:1126-1132. doi:10.1016/j.patcog.2008.08.028.

6. Jaffe MJ. Chapter 9: Mammography.

7. Wael Abdel-Rahman Mohamed Ahmed. COMPUTER AIDED DIAGNOSIS OF DIGITAL COMPUTER AIDED DIAGNOSIS OF DIGITAL. 2009;(December).

8. Koukou V, Martini N, Fountos G, et al. Nuclear Instruments and Methods in Physics Research A Dual energy subtraction method for breast calci fi cation imaging. Nucl Inst Methods Phys Res A. 2017;848(December 2016):31-38. doi:10.1016/j.nima.2016.12.034.

9. Mansour SM, Adel L. Characterization and guided-procedures of breast suspicious microcalcifications: can MicroPure ultrasound do it ? Egypt J Radiol Nucl Med. 2012;43(3):499-505. doi:10.1016/j.ejrnm.2012.07.001.

10. Khan FM. Physics of Radiation Therapy Third Edition. J Am Med Assoc. 2003:1138. doi:10.1001/jama.1953.02940340098036.

11. MURTY RC. Effective Atomic Numbers of Heterogeneous Materials. Nature. 1965;207(4995):398-399. doi:10.1038/207398a0.

12. Kurudirek M. Effective atomic numbers and electron densities of some human tissues and dosimetric materials for mean energies of various radiation sources relevant to radiotherapy and medical applications. Radiat Phys Chem. 2014;102(May):139-146. doi:10.1016/j.radphyschem.2014.04.033.

13. Mobley E. Detecting Microcalcifications in Digital Mammograms with ImageJ. 